\title{
Predictors of psychological well-being among Malaysian graduates
}

\begin{abstract}
Investigations in the field of psychology have traditionally paid attention to studying mental health problems and their prevention (Kaplan, Shema, \& Leite, 2008; Kokko, Korkalainen, Lyyra, \& Feldt, 2012). However, a lack of psychological problems is not necessarily an indicator of the psychological well-being of individuals. Therefore, this study is an attempt to investigate the extent to which the components of cognitive emotion regulation, social support, and physical activity influence the psychological well-being of graduate students in a Malaysian university (University Putra Malaysia). A total of 534 graduate students were selected from this university and the sample size was determined by proportional sampling. Data was analyzed using the Structural Equation Model. The findings of the study revealed that the psychological well-being of Malaysian graduate students was significantly influenced by planning, catastrophyzing, significant others' support, reappraisal, other-blame, selfblame, friend support, putting into perspective, acceptance, and walking. Among these, it appeared that planning was the main strategy that influenced the psychological well-being of the Malaysian graduate students in this study.
\end{abstract}

Keyword: Psychological well-being; Cognitive emotion regulation; Social support; Physical activities 